\title{
Bit Error Performance of Variations along the Spectrum for the Several Sub Carrier
}

\author{
Sudhamathi. K,Deepa, S.Sabapathy, Ananthi' Krishnasamy ,Padmanabhanand, J.Rama
}

\begin{abstract}
Present scenario of mobile communication is to meet the demands of Error free and high data rate communication. This can be achieved with multi carrier modulation technique. In Multi carrier modulation, the transmitting data is split into several components which is modulated and transmitted with different carriers which leads to less Inter Symbol Interference, multipath fading and impulse noise. A novel method of multi carrier modulation and demodulation was done by multiplying user symbols with several sub carriers then combining the several modulated signals and transmitted over a noisy channel. Analysis was carried out after demodulation by comparing received symbols with transmitted symbols. Simulation was carried out to analyze the Bit error rate (BER) performance of multi carrier modulation system as a function of Number of sub carriers and spacing between them. It is found that BER performance is not consistent for all sub carriers. BER performance degrades as sub carriers increases from 1 to $n$, where $n=f s / 2$. It is also observed that the BER performance is approximately stable throughout the specified spectrum for a given sub carrier.
\end{abstract}

Keywords-Bit Error Rate, Filter Bank Technique, Multi Carrier Modulation Technique

\section{INTRODUCTION}

The era of digital communication by telephone lines began in the eighties of the last century, employing phase shift keying in the V32 bits modems with bit transfer rate serially at 2400 to 9600 baud. A phase shifted sine wave analog signal could convey one data bit in Binary Phase shift keying (BPSK), four in Quaternary Phase Shift Keying (QPSK) and more bits with amplitude adjustment in addition to phase. However, it was not possible to increase the bit rate to higher values as they are now, because of the processing difficulties at higher frequencies. Processing at the receiver end would involve computation to retrieve the bit values and in the 90's also, the speed of ordinarily available microcontroller chips could not meet the time constraints. Since the late 90's and from then on, the veteran communication product giants like Motorola, Qualcomm, Broadcom and others have made rapid strides with the more and more availability of very high speed processors, ADCs and DACs. Frequencies have increased from the kilo range to mega cycles range over the years.

Revised Version Manuscript Received on Jun 20, 2019.

K.Sudhamathi, Dept. of NS \& IT, University of Madras, Chennai, India.

A.Deepa, Dept. of NS \& IT, University of Madras, Chennai, India.

K.Padmanabhan, Dept. of ECE, MVJ College of Engineering, Banglore, India.

S.Ananthi, Emeritus Professor, Anna University, Chennai.

J. Rama, Dept. of NS \& IT, University of Madras, Chennai, India
Wireless communication prevailed in the mid 90's and cellular telephony using low microwave frequencies and short distance repeaters made it possible to transmit voice and data from handsets to base stations and vice versa. With an R.F. carrier at about $1 \mathrm{GHz}$, the modulating frequencies could be in the $10 \mathrm{MHz}$ range.

Digital data in binary digits (bits) are represented by sine wave patterns. The phase of the wave varies with the data bits or symbol. A sine wave needs at least two points on it and that means the sampling rate divided by two will be the maximum rate of symbol transmission.

If it were not for the developments made by the microprocessor and DSP componentsindustry, the possibility of the evolution of the said standards would have been delayed verymuch. We will not be having thebluetooth or WiFi until after another decade. Thecombination of several subcarrier signals was thought to be best achieved by using theomplex procedure of the Fourier Transform. In fact,the method uses an inverse Fast Fouriertransform (IFFT) algorithm, as many times a second as the data throughput demands. It isclear that very fast FFT routines has to be included in the firmware of the modem. In a ratherhasty and ambitious approach, the developments that have been possible to today's standardshaveutilised the expertise of the vendors making processors and the people who developedthe firmware programs.

To meet the demands of high data rate such as video calls, video conferencing, high speed internet access in wireless indoor and cellular communication "Multi carrier modulation" (MCM) technique is a key for $4^{\text {th }}$ and future $5^{\text {th }}$ generation cellular communication [1]. In Multi carrier modulation, the transmitting data is split into several components which are modulated and transmitted with different a carrier which leads to less Inter Symbol Interference, multipath fading and impulse noise[2].

With respect to bit errors in multi-carrier communication, there is scope for considerable improvement in performance by proper selection of subcarriers for dense data modes. Even in DSL broadband, there are certain subcarrier channels which are better disposed than the rest with regard to performance. Thus, a study of the relative error vulnerability of the subcarrier channels among themselves is a fruitful study of pursuit to exploit the best data rates with the several subcarriers. Therefore, the paper shows calculated results of several choices of subcarriers and the bit errors in each of them for random data at different signal to noise ratios. The results indicate which of the subcarriers are less prone to errors relative to the rest. 
It came to be realized as the speed race started increasing beyond $1 \mathrm{MHz}$ and upwards, that by sending data at such frequencies gave rise to problems at the receiver end and error percentage sprang up to unacceptable levels due to several causes, even with redundant data packets using convolution encoding method and decoding complexity involving the Viterbi's algorithm.

It came to be realized as the speed race started increasing beyond $1 \mathrm{MHz}$ and upwards, that by sending data at such frequencies gave rise to problems at the receiver end and error percentage sprang up to unacceptable levels due to several causes, even with redundant data packets using convolution encoding method and decoding complexity involving the Viterbi's algorithm.

This led to the evolution of using multiple subcarrier frequencies within the range of the maximum frequencies of 1,10 to $20 \mathrm{MHz}$. Such a splitting of bandwidth and using multiple data symbols on several subcarriers was able to a single symbol at the fsr/ 2 rate. In other words, multi carrier modulation does not increase the throughput as is misconceived by many. The best among the early date publications on the use of multiple subcarriers for modulation can be stated as that of John Bingham, in 1990.The first chapter cites the various aspects of Bingham's IEEE paper. At his time, Bingham could not

telephony, Internet standards and the various short range fast data transfer methods in the 2.5 and $5 \mathrm{GHz}$ range. As and when some new developments have been reported, the IEEE formulated standards for the implementation methods of them. The several publications which led to these standards in the IEEE 802.xx versions are to be cited and summarized so that the reader could appreciate the really fast developments which have taken place within a decade.

If we send the data symbol by converting it into sinusoidal waves at the sampling rate frequency of fsr/2 with just two points in the wave, we get the highest data rate, called the Nyquist rate. We could send a symbol with more points on the wave; that would take more time to send a symbol and the rate is less.

With the desire to increase the rate much higher, work has progressed through the efforts of manufacturers of VLSI and ASIC components providing very high speed analog digital and digital analog converters to enable 20, 40 and even 80 mega samples per second.

The paper is organized as follows. The details of Multicarrier modulation schemes are discussed in section I. After a qualitative discussion on OFDM in Section II, the multi-carrier modulation technique with separately modulating sub carriers with sequential decoding ad error performance is described in section III. The next section gives the details of simulation of bit error performance for changing the position of sub carriers infrequency space and results presented, which are applicable to all the above schemes. The end results indicate how it is feasible to select subcarriers for dense data with good error vs noise performance.

\section{A. Multi Carrier Modulations (Mcm)}

In multi carrier modulation several frequency components are used to transmit user data. The user data is split into severalgroupsanddigitally modulated with subcarriers. Then the several modulated signals combined and transmitted.

In figure 1, data is modulated with sub carriers fc1, eliminate one of the problems of modulation of a single symbol at very high frequency. The waveforms of the several subcarrier data will be a composite analog signal whose pattern is not any discernible sine wave, but will look like a jumble of sample points joined together by the DAC.

Any attempt to improve the existing modulation schemes will need a study and comparison of the different types of Digital Modulation techniques that are widely used presently in the wireless and wired communication systems. Alternative to sinusoidal modulation, so far not much attempts have been made. The main schemes of modulation are phase shift keying and amplitude variations. So, a review of the schemes starting with Binary Phase Shift keying (BPSK), Quadrature Phase Shift keying (QPSK) and related modulation schemes, followed by the Quadrature Amplitude modulation (QAM) schemes.

In Multicarrier Modulation (MCM), we get the same data rate totally for all subcarriers as the data rate if we send have envisaged the present day very high speed of processing power and memory chip availability at fast speeds and very low economies. Starting with the basic technique of multi subcarrier data modulation, concisely known as MCM, the developments based on this has led to world wide cellular

fc2...fcN with a specified bandwidth having guard band separation to avoid interference. Some of the Multi carrier modulation techniques are discussed below.

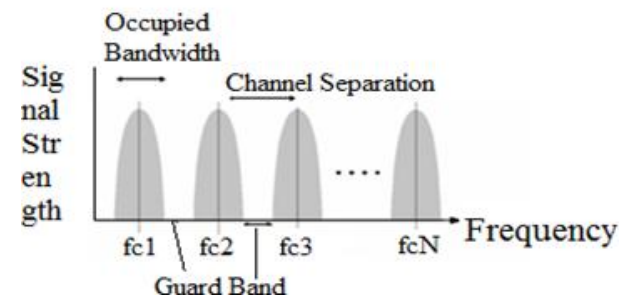

\section{Fig.1. MCM uses Multiple Frequency Bands for Modulation.}

\section{B. Multicarrier Modulation}

Orthogonal Frequency Division Multiplexing (OFDM) is a name given to the multi carrier modulation technique, as described above. In this, if the sampling frequency is say $20 \mathrm{MHz}$ and if 64 subcarriers are used with spacing of $312 \mathrm{KHz}$ between them, data into all these subcarriers will be used to generate sinusoidal waveforms, similar to the figures 2.1 and 2.2. In Fourier transformation, a frequency fc of a subcarrier, when generating a waveform based on the value of the data symbol, the phase being the variable, there will be leakage of the spectrum on the adjacent subcarrier locations in frequency space. This leakage will not interfere with the waveform of theadjacent frequency because of the principle that in decoding, while we use the integral of the product of the reference carrier with the waveform of the subcarrier, the result will be zero, due to the fact that unless $m=n$. So, if $n=m+1$, the result of the leakage of the previous subcarrier will be nil when decoding the next subcarrier. This is how the name "Orthogonal" arose in the definition of the OFDM method. 


$$
\int \operatorname{Sin}(m x) \operatorname{Sin}(n x+\phi)=0
$$

When we use a certain number of subcarriers in the ange 0 to fs, it is advantageous to leave blanks in the beginning and trailing end of spectrum. Other frequencies are used with one symbol per sub carrier maintaining orthogonality among the sub carriers.

Thus in OFDM based communication systems, multiple data symbols are simultaneously transmitted using sub carriers. The high rate data stream of bandwidth $\mathrm{W}$ is divided into $\mathrm{N}$ lower rate data streams and transmitted simultaneously over these orthogonal sub carriers [3]. The individual bandwidth of sub carrier will be $(\mathrm{W} / \mathrm{N})$. The individual bandwidth of sub carrier should be narrower than the coherence bandwidth $(\mathrm{Bc})$ of a channel. The orthogonality among the sub carriers allows overlapping of sub carriers [4]. The orthogonality also ensures the separation of sub carriers at the receiving end. The spectral efficiency is high when compared to Frequency Division Multiple Access systems. OFDM technique is used in the following applications;

1. Digital Audio Broadcasting (DAB)

2. Digital Video Broadcasting (DVB)

3. IEEE802.11 standards Wireless LAN 802.11a,802.11g and

4. IEEE 802.16 WMAX standard 802.16a, e.g.

\section{Essence of multi Carrier modulation Based communication}

When we try to increase the data rate by increasing the frequency of the samples, we get into a problem. For example, when we send samples at $1 \mathrm{MHz}$ rate, each sample time is $1 \mu \mathrm{s}$. There should be at least 8 samples per data symbol, equivalent to a symbol time of $8 \mu \mathrm{s}$. This corresponds to a data rate of $1 / 8 \mathrm{Msymbol} / \mathrm{s}$. (If we used just Binary Phase shift keying, that would amount to $125 \mathrm{kbits} / \mathrm{s}$ ). The time is equivalent to an electromagnetic wave distance of $3 \times 10^{8} \mathrm{~m} / \mathrm{s} \times 8 \times 10^{-6}=2400 \mathrm{~m}$. The wireless path from transmit to receive end might have multiple reflections en route. There may be signals reaching the receiver from several such paths, each with a different total distance. If the distances of the several paths vary by as much as $30 \mathrm{~m}$, the time of reaching of the same signal will vary by $0.1 \mu$ s. This will not cause much distortion in the received signal when the symbol time is $8 \mu \mathrm{s}$.

Suppose we want to increase the data throughput much more by increasing the sampling frequency to $20 \mathrm{MHz}$. That makes the sample time $0.05 \mu$ s. The symbol time will be similarly 8 times this and would be $0.4 \mu$ s. The path difference time of $0.1 \mu$ s now is one fourth of the total symbol time. This is considerably large. It would distort the received signal very much.

Therefore, it is understood that we cannot increase the data rate by merely increasing the sample rate. An alternative to this problem is found in multi carriermodulation.

Let the carrier frequency of the baseband signal be what we want, viz., 20MHz. In this, we can divide several segments of frequencies. Each such segment will be one carrier frequency. Thus, the first carrier frequency may be 0 $-0.5 \mathrm{MHz}$, the second $0.5-1.0 \mathrm{MHz}$ and the last from 19.5 to 20MHz. Thus, theoretically, we can have 40 carriers at

$0.5 \mathrm{MHz}$ spacing. However, the last carriers cannot be utilized because of sampling problems. We may at best have about 32 subcarriers at $0.5 \mathrm{MHz}$ spacing. Each of these carriers can send one data symbol. Thus, the symbols are 32. Therefore the total time of 32 symbols can be much more than with single carrier transmission. All these 32 subcarriers operate simultaneously. The number of samples per symbol can be increased now. In one symbol time, we pack 32 data symbols. Thus, the time per symbol could be 32 times that of the single carrier transmission for the same datarate.

In single carrier, the data rate could not be as high as 10 Msamples per second $(0.1 \mu \mathrm{s}$ sample time and $0.8 \mu \mathrm{s}$ symbol time) because then, the time of each symbol is so low as to disturb the data by even small multiple path time delays of $\mu$ s. But with multiple carriers, the $20 \mathrm{MHz}$ now carries 32 symbols and so, to get the same data rate of 10Msamples/second, we pack 32 in each symbol time, thereby increasing the symbol time from $0.8 \mu$ s to 32 times this value, or $25 \mu \mathrm{s}$. Then, the path reflection time delays are too small to corrupt the data.

\section{OFDM AND ITS QUALITATIVE DESCRIPTION}

What we have gained in OFDM with so many closely spaced sub carriers with data throughput can also be achieved by using less sub carriers with more spacing between them. Spaced subcarriers lend themselves to less errors. Figure 2.15 shows how all the frequency components from 1 to 64 are used as sub carriers in OFDM method of multi carrier modulation technique (IEEE 802.11).

In order to perform such a study, first of all, a set of possible ( frequency division multiplexing (FDM) patterns are to be chosen. That means, in the frequency space, since not all subcarriers are to used (non OFDM), the spacing between the used subcarriers is a vital aspect. Of course, the increase in spacing is going to reduce the data throughput, but at an improved error performance. Since we have introduced the concept of packing more symbols in one and the same subcarrier, throughput reduction is not much compared to OFDM, but the error performance improves considerably.

Therefore, as a first approach to such an analysis, we have taken the following configurations for the FDM spacing patterns. In IFFT/FFT Direct method (OFDM) method, it is possible to have only one symbol per sub carrier. Symbol error rate analysis is done for varying SNR. It is found error performance is better in sequential method and in spaced carrier MCM. Further, in order to elucidate the error variations among the subcarriers with respect to their position in the spectrum, simulated results have been shown. Then, it is shown how the frequency division multiplexing with less subcarriers is prone to less errors than the full spectrum OFDM. It is possible even with less number of subcarriers to attain the full throughput as in OFDM by our method of packing more than one symbol in one subcarrier. With a spacing of four between subcarriers and using symbols per subcarrier as four is the same as full OFDM. 
In OFDM, high rate data is divided into a number of ower rate data (serial to parallel) and baseband modulation is done with different sub carriers which are orthogonal to one another. Cyclic prefix and guard band added to avoid Inter Symbol Interference and Inter Carrier Interference. In OFDM modulation and demodulation can be easily achieved with IFFT / FFT technique [5] as shown in Fig.2a.
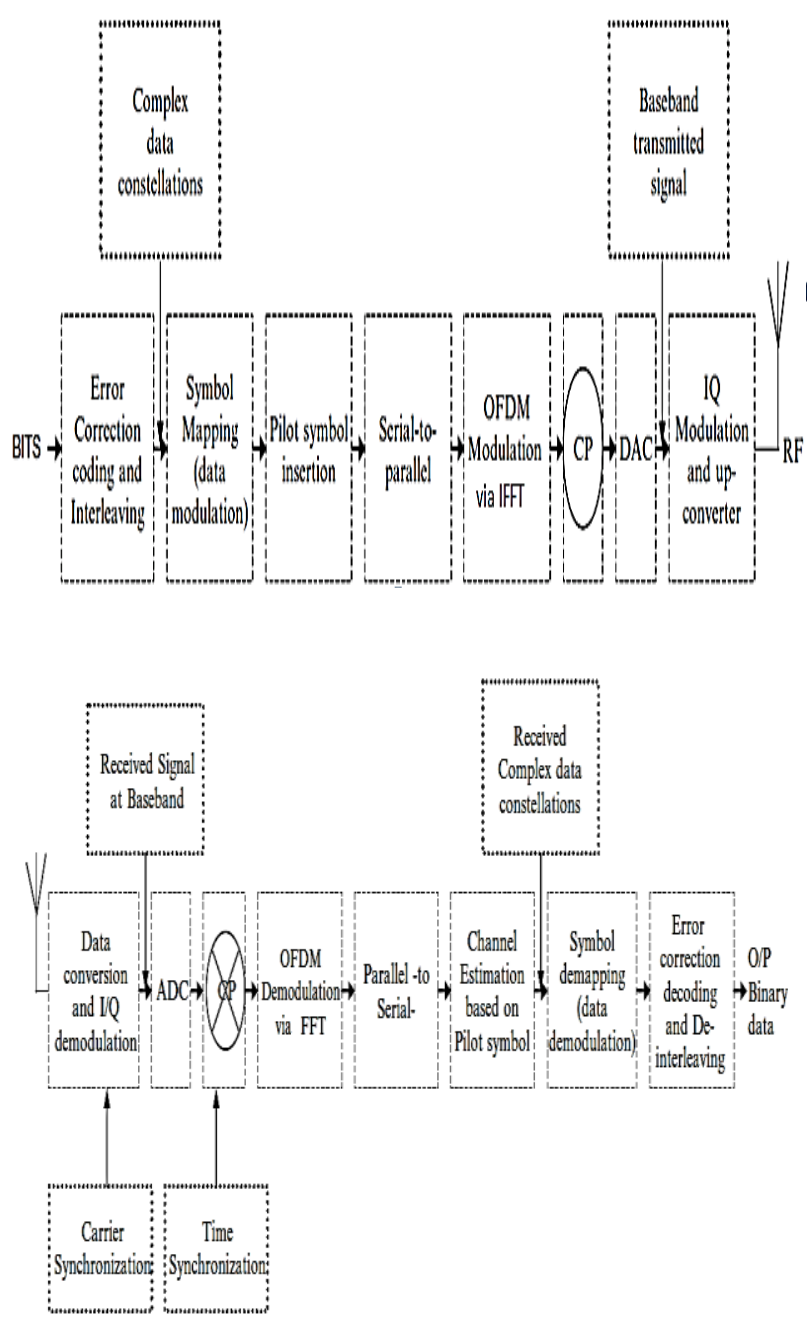

Figure 2.a OFDM Transmitter and Receiver
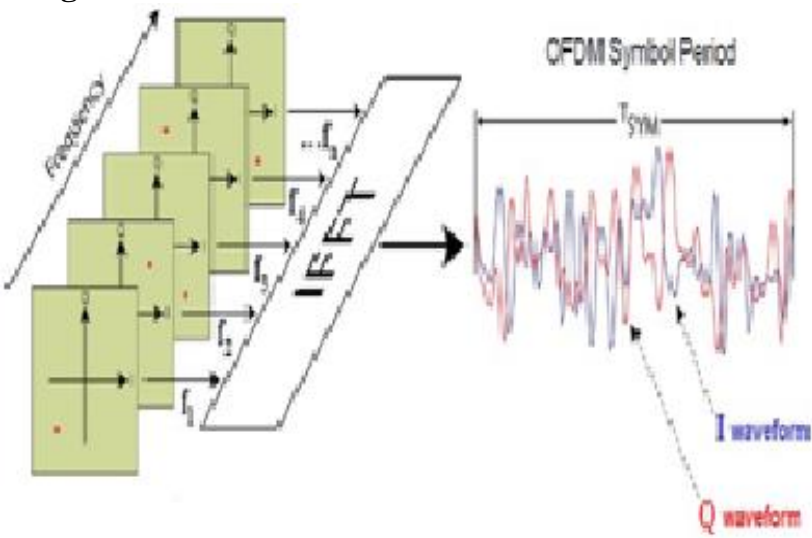

Figure.2b Thephasor values of the several sub carriers can be inverse Fourier Transformed to get the time signal for this particular time slot. This time signal would be used to modulate the R.F. Carrier.

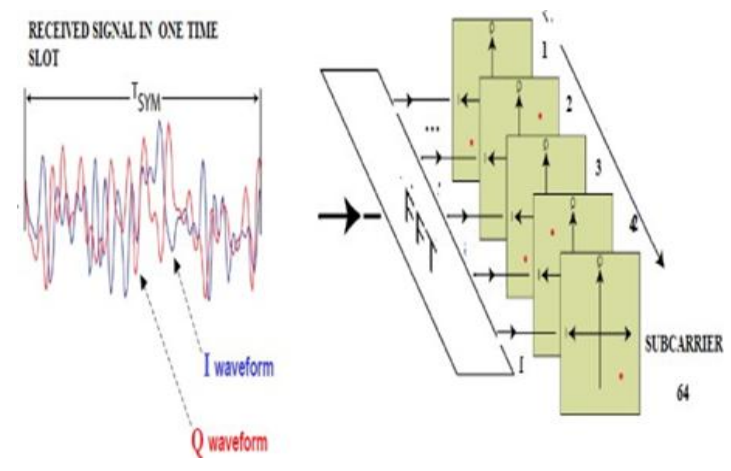

Figure.2c At the receiver end, the analog signals are Digitised and a FFT performed, the several frequency slot real imaginary values are plotted on the several subcarrier constellation complex plots and then their data determined.

In Fig. 2b, this is shown for several subcarriers with the respective frequencies $f_{1}, f_{2}, \ldots, f_{n}$. The combination of these symbols gives rise to the total signal in time encompassing all the symbols. At the receiver, the time signal alone is retrieved via the RF intermediate frequency amplifier. If the real and imaginary parts I and Q are modulated on the high frequency carrier in quadrature, i.e., as the cosine and sine components of such a high frequency sine wave, then we would get back, through the receiver's I.F. amplifier, the same two signal components.

Thus, multi-carrier modulation uses sine modulated signals of frequency multiplexed baseband frequencies. The process is performed digitally by the IFFT and FFT pair at transmitter and receiver (Fig.2c).

The drawbacks of OFDM includes frequency offset and phase noise causing ICI due to non-orthogonally among sub carriers. Its amplitude fluctuations lead to high Peak to Average power ratio [6].

\section{SEPARATE MODULATION FOR EACH SUBCARRIER AND COMBINATION INTOONETIMESIGNAL WITHSEQUENTIAL SUCCESSIVE DECODING}

In this work of multi carrier modulation technique, several user information is taken. The information from several users is first converted to binary form. Then modulate each sub carrier with symbol corresponding toits binary data.Thus analog signal is obtained. Each user information is separately modulated with sub carrier. It is taken care that the modulated analog signal of each sub carrier is of same size so that all the modulated sub carriers can be added for combining as shown in figure 3.b. Figure 3.arepresents total received signal which contains all the sub carriers information. We get phase of a single sub carrier by integrating with respect to that sub carrier. This is achieved using DDEMOD command of MATLAB.

The following is a novel method of demodulation. Edge discontinuities of successive sub carrier waveforms may give rise to errors while doing the demodulation (by integration of product of sub carrier frequency waveforms with the received signal waveform).To improve demodulation due to the presence of such errors, after demodulation of sub carrier $\mathrm{n}$, the sub carrier $\mathrm{n}$ data is again modulated and subtracted from the total received signal voltage. 
This eliminates the possibilities of interference with adjacent signal with the next sub carrier. This can be termed as sequential successive decoding. In FEC convolutional encoding method, there is a decoding scheme called sequential decoding. This is not the same as that. Here we are not taking up any redundant data addition for FEC. We decode one subcarrier after another starting from1 and proceed till the last modulated subcarrier. In this approach, individual sub-carriers performance is measured and analyzed. Matlab computational tool was used to simulate the multi carrier modulation mentioned above.

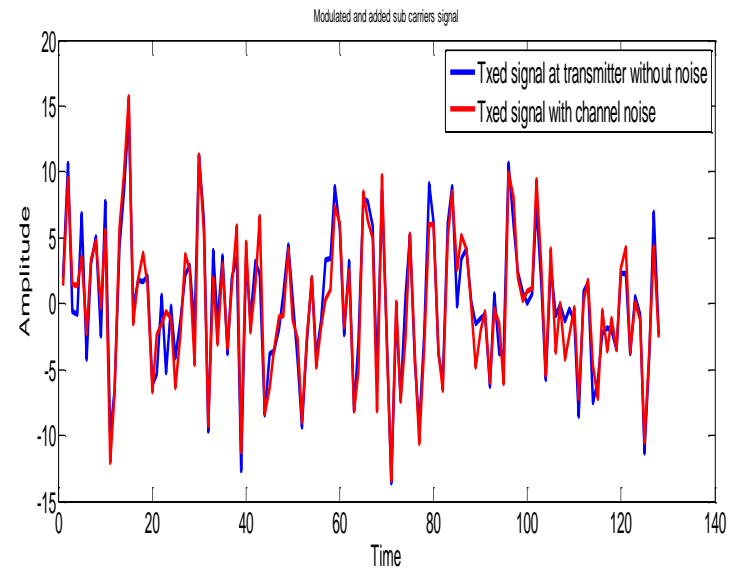

Figure3.a Representing Analog Voltage of PSK Modulated and added subcarriers signal with and without noise

Simulations are carried out to analyse the Bit error rate performance of multi carrier modulation system as a function of sub carriers by varying the sub carrier frequency for a specified spectrum [13].

\section{ERROR DIFFERENCES AMONG THE SUB CARRIERS}

So far there is no literature on this aspect of subcarrier number preferences. Some sub carriers may have less errors and some others have more errors in the presence of noise. The modulated sine waves are just segments of sinusoids having discontinuous edges.

If there is no noise, even with such discontinuities, demodulation process correctly decodes the symbol because of the orthogonality principle. However noise modifies the discontinuities. Error occurs becauseorthoganality is disturbed. Discontinuity patterns vary among the subcarriers. So it is worthwhile to study if there is considerable difference in the symbol errors among the several subcarriers. Such a study would result in a plot of sub carrier position in $\mathrm{X}$-axis and symbol errors in $\mathrm{Y}$-axis for different noise values. Since MCM would generally devote subcarriers to different users, such a result would indicate how certain users may be at a disadvantage with respect to others.

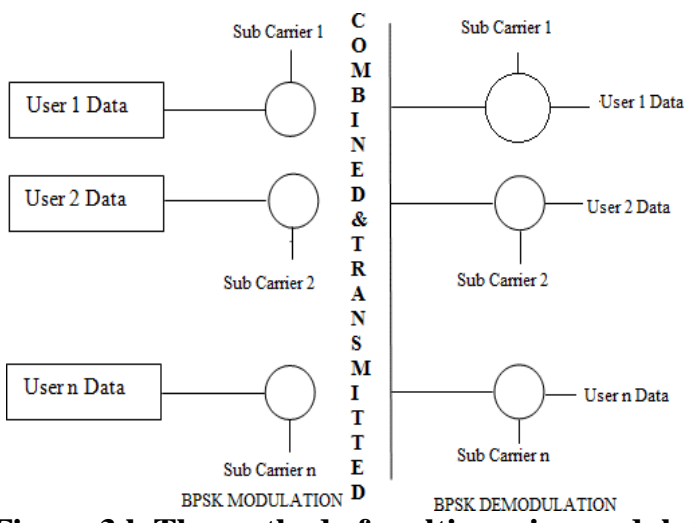

Figure.3.b The method of multi carrier modulation with separately modulated and added subcarriers.

In order to perform such a study, first of all, a set of possible ( frequency division multiplexing (FDM) patterns are to be chosen. That means, in the frequency space, since not all subcarriers are to used (non OFDM), the spacing between the used subcarriers is a vital aspect. Of course, the increase in spacing is going to reduce the data throughput, but at an improved error performance. Since we have introduced the concept of packing more symbols in one and the same subcarrier, throughput reduction is not much compared to OFDM, but the error performance improves considerably.

Therefore, as a first approach to such an analysis, we have taken the following configurations for the FDM spacing patterns.

\section{i) Spacing of four frequency slots between the data encoded subcarrier positions in the frequency space}

Analysis was done for selective frequency components as sub carriers. 15 sub carriers are used to modulate data encoded symbols. The position of first sub carrier was 4 , second sub carrier was 8 and so on with a spacing between sub carrier frequency components as 4 . In all the 15 sub carriers four data symbols are encoded. The same is as shown in figure 3.c.

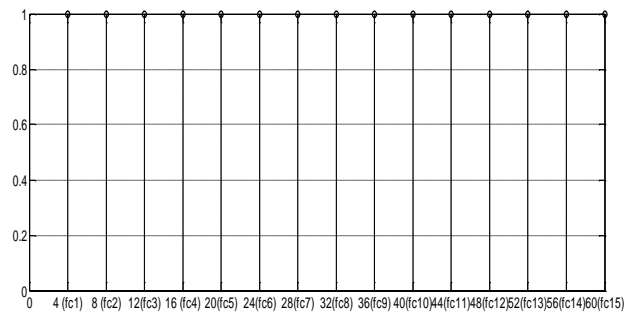

Figure 3.c Representing selective frequency components used for sub carriers 1 to 15 in the given frequency space (QPSK symbols)

\section{ii) Spacing of seven frequency slots between the data encoded subcarrier positions in the frequency space;}

In this configuration, nine sub carriers are used for modulating data encoded symbols. The nine sub carriers are chosen in such a way that spacing between the subcarriers is 7. The position of first sub carrier is at frequency slot 4 . The second sub carrier position is at frequency slot 11 and so on. Thus position of ninth sub carrier is at frequency slot 60 as shown in 
figure 3.d. For this spacing of seven, data symbols are sent through the selective sub carriers and error performance is analyzed by varying SNR.

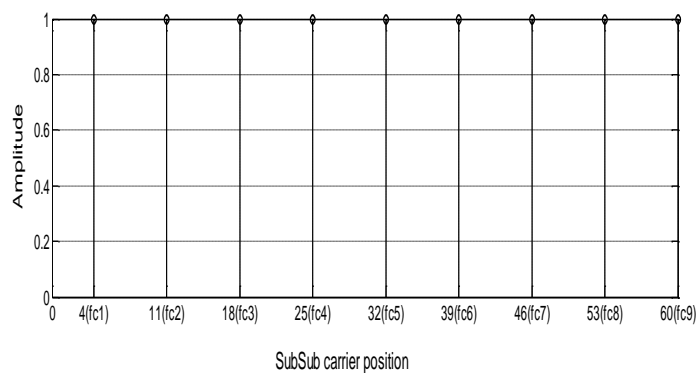

Figure 3d Representing selective frequency components used as sub carriers 1 to 9 in the given frequency space (Spacing 7).

iii) Spacing of nine frequency slots between the data encoded subcarrier positions in the frequency space

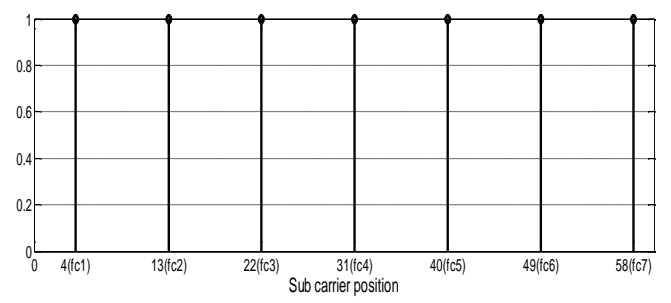

Figure 3e Representing selective frequency components used as sub carriers 1 to 7 in the given frequency space (Spacing 9)

In this configuration seven sub carriers alone are used for modulating data encoded symbols. The seven sub carriers are chosen in such a way that spacing between the subcarriers is 9 . The position of first sub carrier is at frequency slot 4 . The second sub carrier position is at frequency slot 13 and so on. Thus position of seventh sub carrier is at frequency slot 60 as shown in figure 3.e. For this spacing of nine, data symbols are sent through the selective sub carriers and error performance is analyzed by varying SNR. In all the above studies $\mathrm{N}$ has been chooses as 64 . This is without less of generality.

The simulation was done with random QPSK symbols of several hundreds and the noise level was varied from 1 to 10 value. The bit errors were collected and noted for the various SNR values. Number of sub carriers is varied as 15 , 9 and 7. As number of subcarriers increases, spacing between the sub carriers in the given frequency spectrum decreases. Simulations were carried out for sub Carrier spacing of 4, $7 \& 9$ with numbers of subcarriers as 15, 9 and 7 for 50000 symbols per sub carrier. Sampling Frequency $F_{s}$ $=128$, Frequency spectrum used from 1 to 64, Symbol frequency $F_{d}=4$, No of waves per symbol $=F_{c} / F_{d}$, Total No of waves $=\left(\mathrm{F}_{\mathrm{c}} / \mathrm{F}_{\mathrm{d}} *\right.$ No of Input symbols $)$.

For 15 sub carriers, with sub carrier spacing of 4, SNR was varied from 1 to $10 \mathrm{~dB}$. Maximum percentage of bits in error occurs at $1 \mathrm{~dB}$ is $34 \%$. It is found that number of errors is not same for all the sub carriers. It is minimum at the ends of frequency spectrum and increases at some sub carriers and decreases at some sub carrier positions. The maximum dips ss SNR is increased; Error bits decrease as shown in figure 3f.

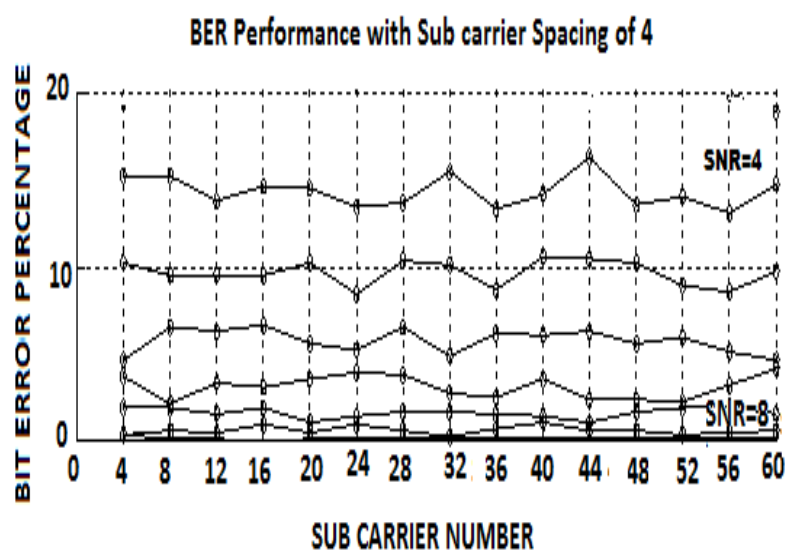

Figure 3f BER performance with 15 Sub carriers spacing of 4 for varying SNR 1:10dBY-axis is \% error

For 9 sub carriers with sub carrier spacing of 7 maximum percentage of bits in error occurs at $1 \mathrm{~dB}$ is approximately $10 \%$. Its performance shown in figure $3 . \mathrm{g}$

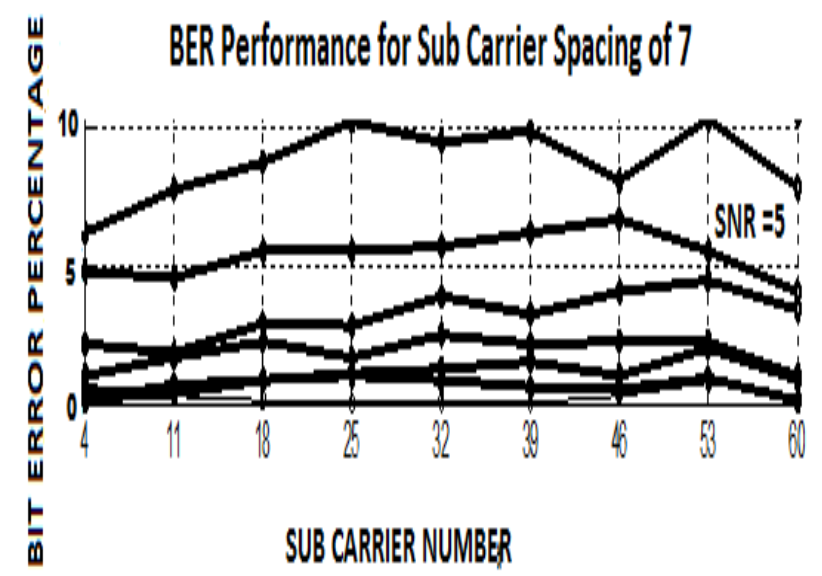

Figure 3g BER performance with 9 Sub carriers spacing of 7 for varying SNR 1:10dBY-axis is \% error bits out of total bits sent.

For 7 sub carriers with sub carrier spacing of 9 the maximum percent of bits in error occurs at $1 \mathrm{~dB}$ is approximately $8 \%$. Its performance shown in figure $3 . \mathrm{h}$.

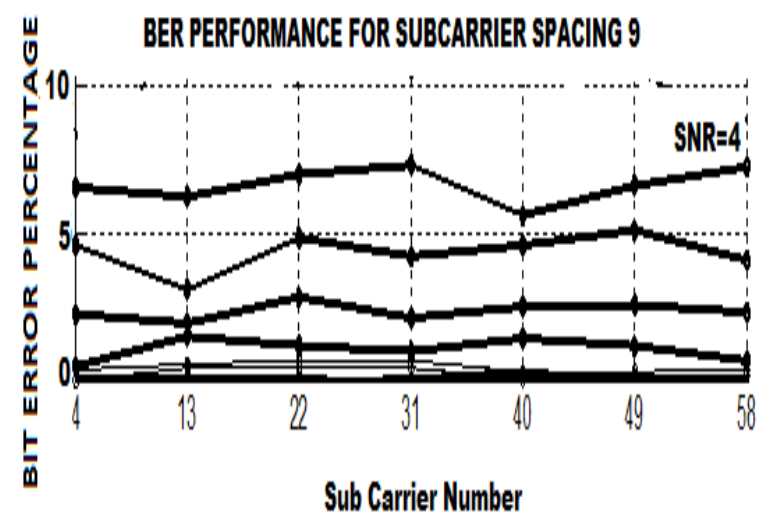

Figure 3h BER performance with 7 Sub carriers spacing of 9 for varying SNR 1:10 dB 
It is found as the spacing between the subcarriers increased; the no of bits in error is reduced.

\section{CHANGING SUB CARRIERS POSITION IN THE FREQUENCY SPACE AND ANALYZING ERROR PERFORMANCE}

In order to estimate errors when the subcarriers position is shifted in the frequency spectrum, simulations were carried out with just 5 sub carriers (QPSK) transmission. Noise was added and received. The five sub carriers frequency positions occupy the frequency spectrum from 1 to 5 or 6 to 10 or 11 to 16 etc. over the total sampling frequency of 1024.

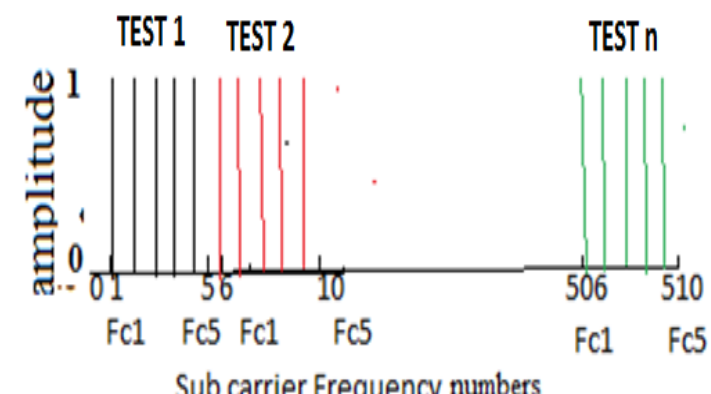

Figure 4a Varying Sub Carriers Position from 1 to 512 in segments of 5 subcarriers.Only 5 subcarriers are data loaded, but these are at different positions in frequency space.

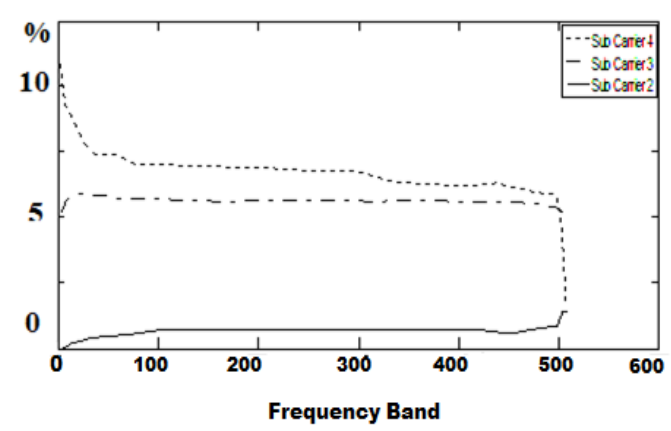

Figure 4b Error percentage with 5 sub carriers at positions from 1 to 510 (fs= 1024). SNR = $5 \mathrm{~dB}$. Plot shows the carriers 2,3 , and 4 only.

The number of data was 4 for each subcarrier in QPSK 2 bit format. 100 random data were simulated and averaged shown in figure 4.a. The bit error was collected for each sub carrier. A plot of each subcarrier frequency and its error bits was made at the various positions of test 1 to test $n$.

Subcarriers 1 and 5 show approximately no errors in the received symbols. The middle subcarrier 2 to 4 among the five have errors due to the juxtaposition of adjacent data waveform discontinuities as shown in figure 4.b. The same was inferred for $7 \& 10$ numbers of sub carriers. When the subcarrier centre position was moved along the spectrum, we find that the bit errors are not varying considerably among the various positions.

This simulation is intended to bring out the fact that, unlike what is usually conjectured that the high end frequency subcarriers are supposed to beget higher errorsis not true. Therefore, subcarriers at the lower end of the spectrum as well as those at the upper end all meet the same amount of errors. The errors arise due to the discontinuities in the modulated sine wave patterns. So, irrespective of the frequency position, these discontinuities will disturb the demodulated values. Further, as noted above, in the bunch of five subcarriers alone being used for this test simulation, the end subcarriers have nil errors even for this high $\mathrm{SNR}=5 \mathrm{~dB}$ value. That is because the discontinuities are absent at least on one side for the end subcarriers in this five group bunch.

\section{CONCLUSION}

In this paper, two methods of MCM were discussed. They are IFFT/FFT Direct method (OFDM) and sequential successive demodulation method. In the first method, it is possible to have only one symbol per sub carrier. In the second method, it is possible to send one or more symbols per sub carrier. Symbol error rate analysis is done for varying SNR. It is found error performance is better in sequential method and in spaced carrier MCM.I

Further, in order to elucidate the error variations among the subcarriers with respect to their position in the spectrum, simulated results have been shown. Then, it is shown how the frequency division multiplexing with less subcarriers is prone to less errors than the full spectrum OFDM. It is possible even with less number of subcarriers to attain the full throughput as in OFDM by our method of packing more than one symbol in one subcarrier. With a spacing of four between subcarriers and using symbols per subcarrier as four is the same as full OFDM. By the method of sequential one by one demodulation of the received data into the subcarrier positions, it has been possible to accommodate more than one symbol in a subcarrier. This method does not need any Fourier transform calculation and can be managed by look up table values addition.

\section{REFERENCES}

1. S. Hassan, "Multicarrier Communication Systems with examples in Matlab -A New perspective", Auerbach Publications, Taylor and Francis Group, USA,2016.

2. A.C.Bingham, "Multicarrier modulation for data transmission. An idea whose time has come", IEEE Communication Magazine, 1990.

3. S. Hara and R. Prasad, "Overview of Multi carrier CDMA", IEEE Commn. Mag., Vol. 35, No. 12, pp126-133,1997.

4. W.C.Jake, "Microwave Mobile communications", New York: Wiley, 1974.

5. R.Saltzbery,"Performanceofanefficientparalleldatatransmission",IEEE Transaction on Communication Technology, Vol. 15, No. 6, 1967.

6. N.Yee, J.P. Linnartz and G.Fettweeis, "Multi-carrier CDMA in indoor wireless radio networks", IEICE Trans. Commn, Vol. 77, No. 7, pp. 109-113,1994.

7. S. Rappaport, "Wireless Communications: Principle and Practice", Prentice Hall, 1995.

8. K.Sudhamathi, S.Ananthi and K.Padmanabhan, "Performance Analysis of MC-CDMA System with Trellis based Modulation in a Radio Channel", JI of Instrumentation Society of India, Vol. 46, No. 1, pp. 52- 56,2016.

9. http://wcsp.eng.usf.edu/OFDM_links.html

10. Q.Shi and M. Ltvaaho, "Performance analysis of MC-CDMA in Rayleigh fading channels with correlated envelopes and phases", In:IEE Proceedings - Communications, Vol. 50, No. 3, pp. 214 220,2003 .

11. T.LajosHanzo, B. Muzster, J. Choi, and Thomas Keller, "OFDM and MC-CDMA for Broadband Multi-User Communications WLANs and Broadcasting", Wiley-IEEE Press, Sep.2003. 
12. http://www.nutaq.com/blog/filter-bank-multicarrier-fbmc$\% \mathrm{E} 2 \% 80 \% 93$-potential-concept-5g-

13. B.F. Boroujeny, "OFDM VersusFilterBank Multicarrier", Signal Processing Magazine, IEEE, Vol. 28, No. 3,pp. 92-112,2011.

\section{AUTHORS PROFILE}

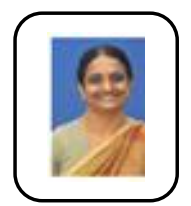

Ms.K.Sudhamathi,did her BE in ECE from Sri Venkateswara College of Engg.,Chennai and ME( Communication Systems) from College of Engg,Guindy,Chennai. Presently doing her Ph.D.in University of Madras in the field of baseband digital modulation.Her areas of specialization range from Microprocessors and Microcontrollers and, Telecommunications.

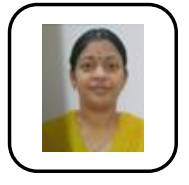

Ms.A.Deepa, did her PG in Seethalakshmi Ramaswami College. She continued her M.Phil., in Bharathidasan University. She had cleared her NET in 2013. Presently doing her Ph.D. in University of Madras. Her areas of specialization includes Microcontroller Applications, Adv. Communication and Colour detection techniques.

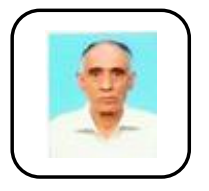

Dr.K.Padmanabhan, did his Grad.Brit.IRE, B.E. from Guindy Engg.College and Doctorate from the Madras University and has served as Professor and Head of the Instrumentation Centre, University of Madras. After retirement, he is A.I.C.T.E. Emeritus Professor in the Anna University. He is a Fellow of IETE,IEE and Sr. Member IEEE. His areas of specialization range from Applied Electronics, Microprocessors, Telecommunications and DSP.

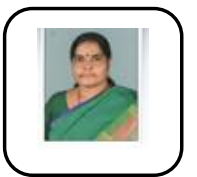

Dr.S.Ananthi, got her B.E. in ECE from the Anna University. She did M.Tech. at the Indian Institute of Science and later did Doctorate from University of Madras. She worked in the University as Professor and Head $\mathrm{i} / \mathrm{c}$ of NSIT department till June 2018.After retirement, she is Professor, ECE in MVJCE. Her areas of specialization includes in DSP, Adv. Communication and Biomedical telemetry. She was awarded the Env. TN award for the year 2004.

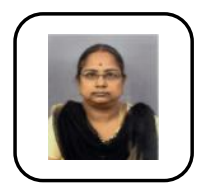

Mrs. J. Rama, did her BE in ECE from ACCET, Karaikudi and ME (Instrumentation and Control Engineering) from MIT, chennai. Currently she is the Principal in the Government polytechnic as well pursuing her $\mathrm{Ph}$. D under the guidance of Dr. S. Ananthi, Department of Networks and Information Technology, University of Madras. Her areas of specialization includes in DSP, Adv. Communication and Microcontroller applications. 